\title{
High-Resolution SEM Imaging with Aberration Correction for Highly Precise Measurement of Semiconductors.
}

\author{
M. Konno, ${ }^{*}$ Y. Suzuki, ${ }^{*}$ H. Inada, ${ }^{* *}$ K. Nakamura**
}

* Global Application Center, Naka Division, Nanotechnology Products Business Group, Hitachi High-Technologies, 11-1, Ishikawa, Hitachinaka, Ibaraki, 312-0057, Japan

** Advanced Microscope System Design $2^{\text {nd }}$ Department, Naka Division, Nanotechnology Products Business Group, Hitachi High-Technologies, 882, Ichige, Hitachinaka, Ibaraki, 312-8504, Japan

The manufacturing of semiconductor devices requires highly precise measurement a film thickness, such as a gate oxide layer and barrier metal layers with atomic resolution using transmission electron microscopy. However, recent improvements in semiconductor transistors become led to a reduction gate dimensions bellow $20 \mathrm{~nm}$, in which precise site-specific sample preparation that requires much time leading decreased throughput. In order to reduce the loads placed on sample preparation, we have developed a high-resolution secondary electron (SE) imaging technique using the dedicated STEM instrument with a high efficiency secondary electron detector, Hitachi HD-2700 [1].

The incorporation of the high efficiency secondary electron detector makes high resolution imaging of semiconductor specimens while avoiding the time consuming sample preparation required to produce thin sections for TEM. The HD-2700 is equipped with a CEOS GmbH aberration corrector and can routinely achieve $1 \AA$ spatial resolution in secondary-electron (SE) imaging and STEM imaging [2]. Here we report an application of this new technology for high accuracy critical dimension measurements of semiconductor materials.

Figure1 illustrates the overall process used for STEM observation of semiconductor materials. First, a small section (Size: $12 \mu \mathrm{m}(\mathrm{W}) \times 7 \mu \mathrm{m}(\mathrm{H}) \times 3 \mu \mathrm{m}(\mathrm{D})$ ) of the semiconductor transistor is extracted from the bulk sample and mounted on the specimen stub inside of the FIB specimen camber using the NB5000 FIB-SEM equipped with micro-sampling system [3]. Since the reduction of FIB damage is one of the most important issues for to obtaining quality SE images, final milling of the sample was carried out at $2 \mathrm{kV}$ in which thickness of FIB damage layer was about $3 \mathrm{~nm}$ [4].

Figure 2 shows the angular dependency of SE signal images. Specimen tilt angle was $0^{\circ}$, observed along the $\mathrm{SrTiO}_{3}[001]$ zone axis (a), $2^{\circ}$ (b) and $5^{\circ}$ (c), respectively. Here, atomic column of $\mathrm{Sr}$ and $\mathrm{Ti}$ become invisible by change in tilt angle, and therefore, specimen thickness was $1 \mu \mathrm{m}$ in order to align the crystal orientation using electron diffraction pattern.

Figure 3 shows the cross sectional SE image of semiconductor transistor (a) and the magnified SE image observed along the $\mathrm{Si}[011]$ zone axis (b). The beam current was measured using a Faraday cup in the specimen holder and the beam current was found to be $200 \mathrm{pA}$ using a $0.2 \mathrm{~nm}$ diameter probe and an accelerating voltage of $200 \mathrm{kV}$. Figure 3a clearly shows the semiconductor structures such as a metal gate, high-k gate insulating layer, source and drain with great clarity. In figure $3 b$, the magnified SE image shows that it is possible to resolve the $\mathrm{Si}(111)$ plane, which has a spacing of $0.314 \mathrm{~nm}$. These results demonstrate the ability to perform high precision measurements of crystal lattices for the structural characterization of semiconductor materials. This capability may also be used as a technique for precisely calibrating the magnification settings for STEM instruments.

References 
[1] Inada, H., Wu, L., Wall, J., Su, D., \& Zhu, Y., J. of Electron Microsc. 58, 111-122 (2009)

[2] Zhu, Y., Inada, H., Nakamura, K. \& Wall, J., Nature Mater. 8, 808-812 (2009)

[3] Ohnishi,T. and Ishitani,T., JP Patent 2774884 and US patent 5270552

[4] Konno, M., Suzuki, Y., Inada, H., Nakamura, K., Kimoto, K and Zhu, Y., Microsc. And Microanal. 16, 128-129 (2010).
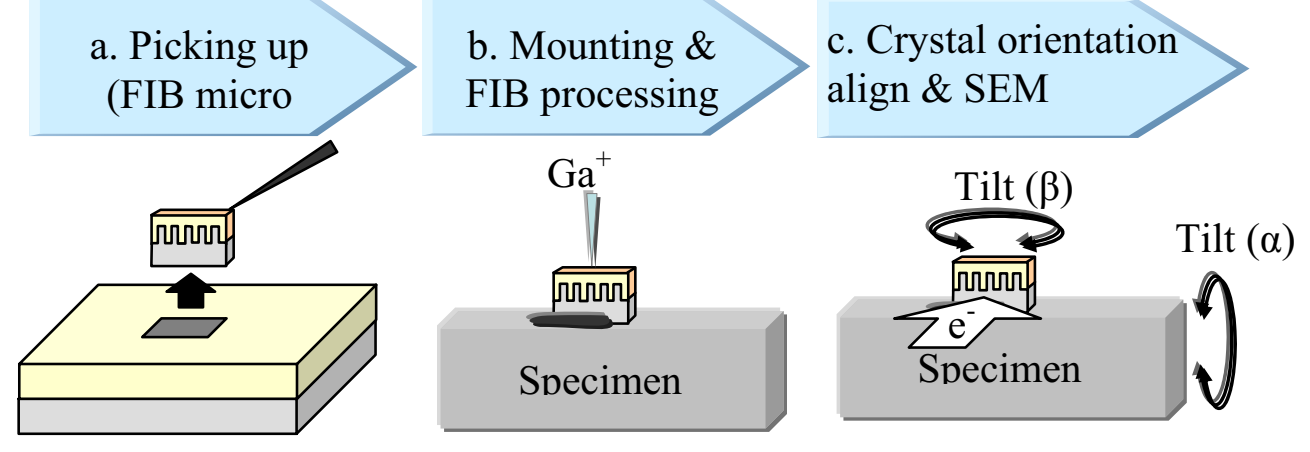

FIG. 1. FIB fabrication and SEM imaging procedure.

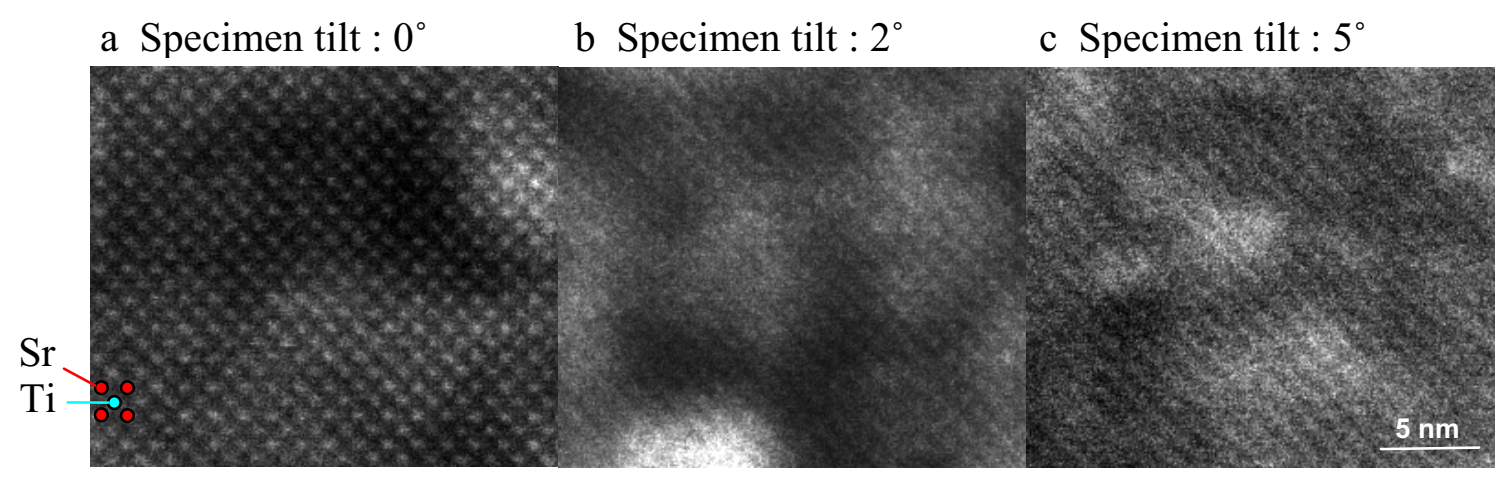

FIG. 2. Angular dependency of high-resolution Secondary electron (SE) images taken with HD2700 at $200 \mathrm{kV}$ along the $\mathrm{SrTiO}_{3}[001]$ zone axis. (Magnification : $\times 10,000,000$ )

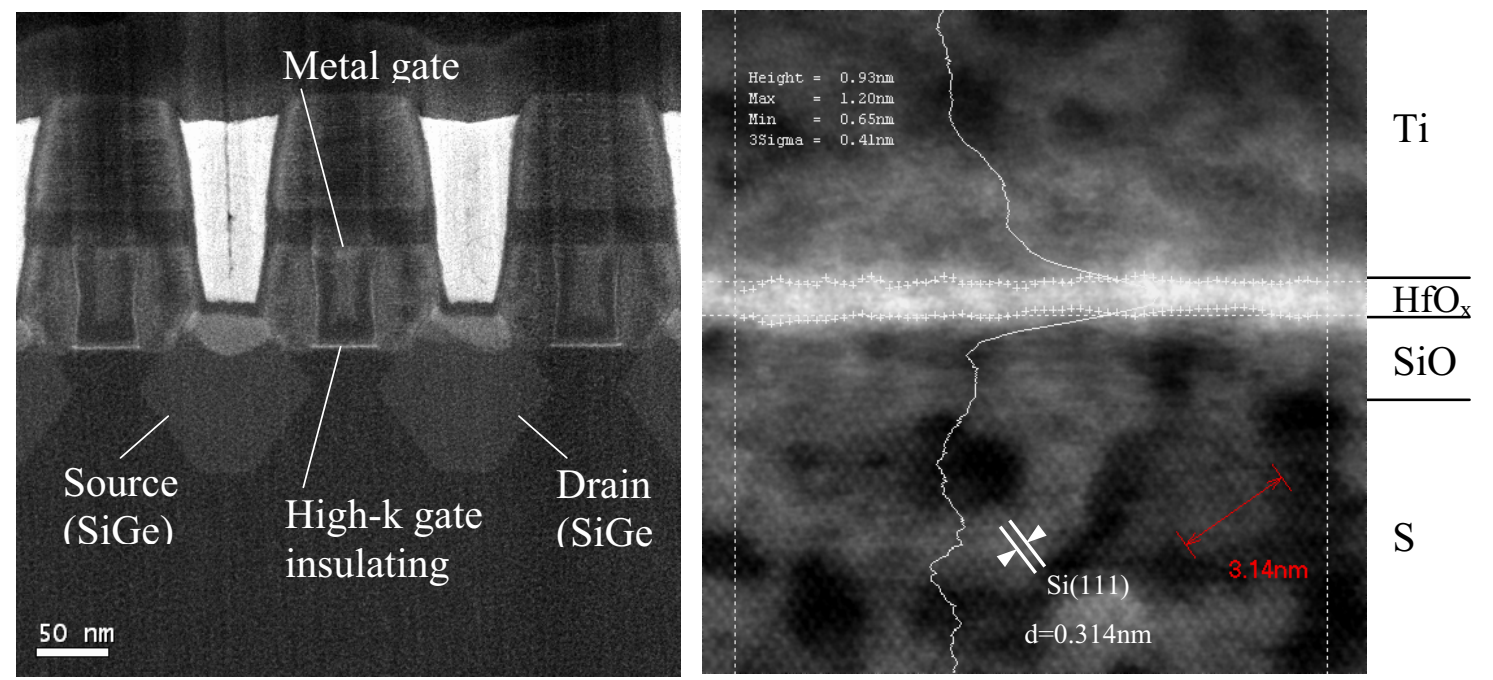

FIG. 3. Overview SE image (a) and magnified SE image (b) of semiconductor transistor taken with HD-2700 at $200 \mathrm{kV}$ along the $\mathrm{Si}[011]$ zone axis. (Magnification a $: \times 180,000, \mathrm{~b}: \times 5,000,000$ ) 